LES ANNALES Les Annales de droit

DE DROIT

12 | 2018

Varia

\title{
Desserte maritime de la Corse et droit de l'Union européenne
}

Les difficultés rencontrées par la Société nationale maritime CorseMéditerranée

Maritime services to Corsica and European Union law. Difficulties faced by the Société nationale maritime Corse Méditerranée

\section{Sébastien Martin}

\section{(2) OpenEdition}

Journals

Édition électronique

URL : http://journals.openedition.org/add/1190

DOI : 10.4000/add. 1190

ISSN : 2606-1988

Éditeur

Presses universitaires de Rouen et du Havre

Édition imprimée

Pagination : 173-185

ISBN : 979-10-240-0937-7

ISSN : 1955-0855

Référence électronique

Sébastien Martin, « Desserte maritime de la Corse et droit de l'Union européenne », Les Annales de droit [En ligne], 12 | 2018, mis en ligne le 05 février 2019, consulté le 05 décembre 2019. URL : http:// journals.openedition.org/add/1190; DOI : 10.4000/add.1190 


\section{Desserte maritime de la Corse et droit de l'Union européenne}

\section{Les difficultés rencontrées par la Société nationale maritime Corse-Méditerranée ${ }^{1}$}

Sébastien MarTiN

La desserte maritime de la Corse est aujourd'hui une compétence de la collectivité territoriale de Corse (CTC). Ce qui est remarquable, bien que tout à fait logique tant le droit européen est devenu primordial, c'est que l'autorité publique ne peut l'organiser sans se conformer au droit de l'Union. De même, les opérateurs présents doivent respecter les règles adoptées par les institutions de l'Union.

En ce qui concerne les liaisons maritimes entre la Corse et le continent, avant que la compétence ne revienne à la collectivité locale, c'est l'État qui, depuis 1976, se chargeait de l'organisation de la desserte. Pour ce faire, il respectait le principe de continuité territoriale qui «commande les modalités d'organisation des transports maritimes et aériens entre l'île et le continent » avec un «objectif de désenclavement ${ }^{2}$ » des régions insulaires qui, pour des raisons géographiques, mais aussi économiques, ne peuvent pas bénéficier de liaisons avec les autres régions ${ }^{3}$.

1. Cette contribution présentée lors du workshop «Insular territories and europeanization » organisé par l'UMR LISA de l'université de Corse, les 9 et 10 novembre 2016, reprend pour partie des éléments présentés par l'auteur le 22 septembre 2015, lors des universités d'été de Bordeaux portant le titre «L'Union européenne et la mer». L’intervention était intitulée «La Société nationale Corse Méditerranée : entre droit du marché intérieur et droit de la concurrence».

2. Maude Elfort, "Continuité territoriale et desserte aérienne: le cas de la Guyane française», JCP A 13 juin 2005, $n^{\circ}$ 24, comm. 1239.

3. Dans ce sens, voir Loïc Grard, «Le droit européen des transports: une référence pour le service public en environnement de concurrence ", dans Jean-Victor Louis et Stéphane Rodrigues, Les services d'intérêt économique général et l'Union européenne, Bruxelles, Bruylant, 2006, p. 387 et suiv. ; «L'Union européenne et le service public, aéronefs et navires dans le même bateau ", DMF 2005, p. 830 et suiv. 
Pour assurer la desserte maritime de la Corse, les autorités n'ont pas choisi la formule la plus courante ${ }^{4}$ et, en l'occurrence, ont délaissé le contrat de délégation de service public (CDSP) au profit de la délégation unilatérale. Toutefois, cela n'est pas si exceptionnel, les délégations unilatérales sont encore utilisées dans le domaine des transports urbains ${ }^{5}$. On soulignera cependant rapidement, car il s'agit d'une situation révolue pour ce qui concerne la Société nationale maritime Corse-Méditerranée (SNCM), que cette attribution directe présente certaines difficultés théoriques à l'égard du droit européen. En effet, la délégation unilatérale est faite, sans mise en concurrence, par une autorité publique au profit d'une entreprise publique qui bénéficie, qui plus est, d'un monopole ${ }^{6}$. À titre

4. À la différence de la délégation contractuelle, la délégation unilatérale présente des limites, en particulier parce qu'il existe certaines restrictions. Une telle mesure n'est notamment possible que si elle est prévue par un texte, voir CE, ass., avis section travaux publics, 9 mars 1995, req. $\mathrm{n}^{\circ}$ 355931, Organisation des transports en région Île-de-France: EDCE 1995, p. 399 et CE, ass., avis section travaux publics, 28 sept. 1995 , req. $n^{\circ} 357262$ et 357263 , Champ d'application de la loi du 29 janvier 1993 au secteur du gaz et de l'électricité, EDCE 1995, p. 402. Dans un arrêt récent, la haute juridiction administrative rappelait d'ailleurs que «lorsque des collectivités publiques sont responsables d'un service public, elles peuvent dès lors que la nature de ce service n'y fait pas par elle-même obstacle, décider de confier sa gestion à un tiers, qu'à cette fin, sauf si un texte en dispose autrement, elles doivent en principe conclure avec un opérateur [...] un contrat de délégation de service public ou, si la rémunération de leur cocontractant n'est pas substantiellement liée aux résultats de l'exploitation du service, un marché public de service», CE, sect., 6 avr. 2007, req. ${ }^{\circ}$ 284736, Commune d'Aix-en-Provence : Rec. CE 2007, p. 155, concl. François Séners, note Jean-Mathieu Glatt, «Les modalités d'une gestion efficace des activités de service public: de l'habilitation unilatérale à l'habilitation contractuelle», LPA 24 juil. $2007, \mathrm{n}^{\mathrm{o}} 147$, p. 13 et suiv.

5. Ainsi, pour ce qui est de l'organisation des transports en commun d'île-de-France, l'autorité qui en a la charge a pour mission principale de désigner les exploitants de lignes en procédant à leur simple inscription au plan des transports (D. $n^{\circ} 49-1473$, 14 nov. 1949, art. 4 relatif à l'harmonisation et à la coordination des transports ferroviaires et routiers, JO 15 novembre 1949, p. 11104). C'est donc cette autorité qui se charge de l'attribution directe des lignes de transport en commun en Île-de-France, notamment celles de la RATP, en procédant à une délégation unilatérale.

6. Voir Martine Lombard, L'État schizo, Paris, J.-C. Lattès, 2007, p. 209 et suiv. De la même manière Gilles Savary soulignait à quel point «des entreprises publiques françaises, voire certaines administrations publiques, [étaient] devenues de puissants opérateurs sur les marchés ouverts, tant en Europe que dans le monde», ce qui lui permettait de conclure : «On comprend mieux l'incompréhension, quand ce n'est pas l'irritation, de nos partenaires européens et de la Commission européenne à Bruxelles à l'égard de nos résistances à ouvrir le secteur des entreprises publiques industrielles et commerciales à leurs homologues européens" (L'Europe va-t-elle démanteler les services publics?, La Tour-d'Aigues, L'Aube, 2005). Voir aussi Antoine Tabouis et Aymeric Hourcabie, "Notion et régime juridiques de la délégation unilatérale de service public », Contrats-Marchés publ. 2005, nº 6 . 
principal, cette formule présente le défaut d'empêcher des concurrents potentiels d'assurer la prestation de service et, par conséquent, de bénéficier de la libre prestation de service reconnue par les traités ${ }^{7}$.

À la fin du monopole d'une durée de 25 ans, l'État avait abandonné la compétence d'organisation de la desserte maritime de l'île pour la confier aux collectivités corses. En effet, en même temps que les lois de décentralisation relatives aux collectivités de métropole étaient votées, le Parlement offrait «à la région de Corse un "statut particulier" caractérisé par un degré de libre administration un peu plus poussé que celui que le législateur devait conférer quelques mois plus tard aux régions de droit commun ${ }^{8} »$. Ce statut mis en place en $1982^{9}$ a offert une véritable autonomie à la collectivité de Corse qui a profité, en 1991 et en 2002, d'adaptations spécifiques ${ }^{10}$. L'assemblée territoriale de Corse, devenue une collectivité territoriale que d'aucuns qualifieront de sui generis, possède un statut qui tend à la rapprocher des solutions adoptées pour l'outre-mer ${ }^{11}$, mais qui lui permet surtout de bénéficier de compétences plus larges, notamment en matière de service public de transport, que celles dont bénéficient les autres conseils régionaux.

À la fin du monopole, l'état du droit européen avait également évolué avec l'entrée en vigueur, au début de l'année 1993, du règlement européen sur le cabotage maritime ${ }^{12}$. Ce faisant, les autorités françaises ont modifié le cadre juridique applicable aux activités maritimes entre

7. Voir art. 34 et suiv. du TFUE

8. Thierry Michalon, «La République et sa périphérie. La légitimité par la décentralisation? ?, dans La profondeur du droit local. Mélanges en l'honneur de Jean-Claude Douence, Paris, Dalloz, 2006, p. 329 et suiv.

9. Voir L. $\mathrm{n}^{\circ} 82-214,2$ mars 1982 portant statut particulier de la région de Corse (organisation administrative), JO 3 mars 1982, p. 748 et L. $n^{\circ} 82-659$, 30 juil. 1982 portant statut particulier de la région de Corse (compétences), JO 31 juil. 1982, p. 2459.

10. Voir L. $\mathrm{n}^{\circ}$ 91-428, 13 mai 1991 portant statut de la collectivité territoriale de Corse, JO 14 mai 1991, p. 6318 et L. $\mathrm{n}^{0}$ 2002-92, 22 janv. 2002 relative à la Corse, JO 23 janv. 2002, p. 1503.

11. Sur les compétences de la CTC, voir Jean-Paul Pastorel, «Les compétences de la collectivité territoriale de Corse», RFDA 1991, p. 741 et suiv., et «Les nouvelles compétences de la Collectivité territoriale de Corse», RFDA 2002, p. 685 et suiv.

12. Règl. (CEE) $\mathrm{n}^{\circ} 3577 / 92$ du Conseil, 7 déc. 1992, concernant l'application du principe de la libre circulation des services aux transports maritimes à l'intérieur des États membres (cabotage maritime), JO L 364, 12 déc. 1992, p. 7. À partir du $1_{1}{ }^{\mathrm{er}}$ janvier 1993 , la libre prestation des services de transport maritime à l'intérieur d'un État membre (cabotage maritime) s'applique aux armateurs communautaires exploitant des navires immatriculés dans un État membre et battant pavillon de cet État membre, sous réserve que ces navires remplissent toutes les conditions requises pour être admis au cabotage dans cet État membre, y compris les navires 
la Corse et le continent avec la loi de $2002{ }^{13}$, laquelle prévoit que «des obligations de service public [soient] imposées par la collectivité territoriale de Corse sur certaines liaisons [...] maritimes pour assurer le principe de continuité territoriale ${ }^{14}$ ». En d'autres termes, la liaison maritime Corse-Marseille a connu l'évolution classique des modes de gestion des services publics industriels et commerciaux. Il a été mis fin au monopole octroyé par l'État à l'entreprise SNCM, ce qui a, en conséquence, permis à la CTC d'organiser un nouveau service public reposant sur des obligations de service public préalablement définies et sur un contrat de service public. La collectivité décentralisée se voit, par ailleurs, reconnu un véritable pouvoir de décision concernant le choix du prestataire. Néanmoins, elle a choisi la SNCM et sa filiale la Compagnie maritime nationale $(\mathrm{CMN})$ pour assurer des délégations de service public (DSP) de 5 ans sur les liaisons en question ${ }^{15}$. Parallèlement

immatriculés dans le registre Euros dès que ce registre aura été approuvé par le Conseil.

13. Voir L. $\mathrm{n}^{\mathrm{o}} 2002-92,22$ janv. 2002 relative à la Corse.

14. L'art. L.4424-20 du CGCT prévoit que l'office des transports de la Corse, établissement public de la collectivité territoriale de Corse à caractère industriel et commercial sur lequel la collectivité exerce son pouvoir de tutelle, et qui exerce les missions en matière de transport que celle-ci lui confie, "conclut avec les compagnies désignées pour l'exploitation [de ces] liaisons [...] des conventions de délégation de service public » et «répartit les crédits visés à l'article L.4425-4 entre les deux modes de transports aérien et maritime, sous réserve que cette répartition reste compatible avec les engagements contractés dans le cadre des conventions conclues avec les concessionnaires et qu'elle n'affecte pas, par elle-même, l'équilibre financier de ces compagnies ». Art. L.4425-4 CGCT: «L'État verse à la collectivité territoriale de Corse un concours individualisé au sein de la dotation générale de décentralisation de la collectivité territoriale de Corse, intitulé : "dotation de continuité territoriale”, dont le montant évolue comme la dotation globale de fonctionnement. À titre dérogatoire, cette évolution ne s'applique pas en 2009. Ce concours est consacré à la mise en œuvre des dispositions des articles L.4424-18 et L.4424-19."

15. En 2001, la CTC a organisé pour la première fois une procédure de publicité et de mise en concurrence, et a attribué une délégation de service public à un groupement composé de la SNCM et de la CMN pour 5 ans, de 2002 à 2007 . À l'expiration de cette concession et après une nouvelle mise en concurrence, la CTC et l'OTC ont conclu une délégation de service public avec le groupement sortant. Cette convention d'une durée de 5 ans et demi est entrée en vigueur le $1^{\text {er }}$ juillet 2007 et devait expirer le 31 décembre 2013. Un $3^{\mathrm{e}}$ contrat aurait dû s'appliquer à partir de 2014. Toutefois, la délibération par laquelle la collectivité décentralisée approuvait la convention de délégation de service public relative à l'exploitation du transport maritime de passagers et de marchandises au titre de la continuité territoriale entre les ports de Corse et le port de Marseille confiée au groupement d'opérateurs historiques a été annulée par le tribunal administratif (jugement $n^{0} 1300012$ et 1300014, 17 oct. 2013) 
à la mise en œuvre de cette DSP, un certain nombre de compagnies maritimes d'origine française ou européenne ont, conformément à la réglementation de l'Union, demandé à assurer des prestations de cabotage maritime à destination de la Corse ${ }^{16}$.

On trouve donc à partir de ce moment-là, deux types de services maritimes à destination de la Corse. D'une part, les services protégés confiés au groupement SNCM et CMN bénéficiant de droits exclusifs et, d'autre part, les services ouverts à la concurrence pris en charge par différents opérateurs maritimes.

Avant de voir les éléments qui ont soulevé des difficultés, on doit commencer par rappeler deux éléments fondamentaux.

Tout d'abord, le droit de l'Union prend en considération les missions de service public. Ainsi, pour les institutions de l'Union, comme pour les autorités françaises, la desserte maritime de la Corse répond à des besoins d'intérêt général justifiant des mesures particulières pour assurer son bon exercice. La Commission, en $2001{ }^{17}$, a ainsi clairement souligné que «la convention conclue par l'État avec la SNCM [...] visait précisément à remédier à l'insuffisance constatée des services réguliers de transport maritime entre la France continentale et la Corse et à permettre la compensation des coûts liés au déficit enregistré par l'entreprise pour satisfaire ces obligations », ajoutant qu' «il résulte du cadre normatif et conventionnel [...] que la SNCM a été soumise, sur l'ensemble des lignes, à une série d'obligations tenant aux ports à desservir, aux fréquences, aux horaires de départ et d'arrivée, à la typologie des navires, aux tarifs à pratiquer, que cette entreprise n'assumerait pas ou n'assumerait pas

à la demande de la société Corsica ferries France ainsi que de la CGPME-CORSICA. Le tribunal a retenu 5 motifs d'annulation portant respectivement sur l'adoption de la délibération au terme d'un vote par groupe; la mise en place d'un régime d'autorisation préalable; la possibilité donnée à l'OTC de modifier le programme et de limiter les capacités des compagnies maritimes soumises aux OSP; le régime de tarification applicable aux passagers non résidents et au fret roulant; le régime de pénalités applicables en cas de manquement aux obligations de service public. Compte tenu de cette annulation, et tant qu'un nouveau dispositif n'aura pas été adopté, le $1^{\text {er }}$ dispositif des obligations de service public, arrêté par délibération $\mathrm{n}^{\mathrm{o}}$ 01/002 $\mathrm{AC} \mathrm{du} 1^{\mathrm{er}}$ février 2001 continuera donc de produire ses effets.

16. Dans un certain nombre de cas, cela a donné lieu à la conclusion de conventions avec la CTC lorsque l'exécution des obligations de service public permettait d'obtenir une compensation financière. Toutefois, à la différence de la convention de délégation de service public, aucune compagnie ne bénéficiait de droit exclusif, permettant ainsi à différentes compagnies de se faire concurrence.

17. Comm. CE, déc. $\mathrm{n}^{\circ}$ 2002/149/CE, 30 oct. 2001 concernant les aides d'État versées par la France à la SNCM, JO L 50, 21 févr. 2002, p. 66. 
dans la même mesure ni dans les mêmes conditions si elle était mue par son propre intérêt commercial».

Ensuite, le droit de l'Union tient également compte de l'éloignement de certains territoires. Pour cela, il utilise la notion d'ultrapériphéricité qui répond elle aussi à cette volonté de désenclavement. Sa définition est cependant plus précise, car les régions concernées doivent justifier « d'un grand éloignement du continent européen, de contraintes géophysiques et climatiques pesantes (toutes sauf la Guyane sont des îles et ont une petite surface) et de conditions socioéconomiques particulièrement défavorables $[\ldots]$ : une diversification économique très faible orientée vers le tourisme et l'agriculture, un déséquilibre entre les importations et les exportations, un taux de chômage très élevé ${ }^{18}$ » et sont précisément identifiées ${ }^{19}$. On comprend donc que la desserte maritime de la Corse ne puisse bénéficier du régime juridique correspondant dans la mesure où la Corse n'est pas une région ultrapériphérique.

Quoi qu'il en soit, la desserte maritime de la Corse correspond bien à la qualification de service d'intérêt économique général (SIEG). Cependant, cela n'a pas empêché les autorités de relever que certaines modalités de l'organisation du service public étaient illégales.

Avant d'aller plus loin, rappelons que les SIEG désignent les services de nature économique, c'est-à-dire des activités de production ou de commercialisation de services marchands qui répondent à un critère d'intérêt général et que les États membres ou la Communauté soumettent à des obligations spécifiques de service public. La qualification de SIEG n'est pas sans conséquences. En particulier, cela permet de déroger à l'application des règles du droit du marché intérieur et de la libre concurrence. Toutefois, la seule qualification n'est pas suffisante, il faut encore, pour les autorités nationales, démontrer que les dérogations sont nécessaires à la réalisation de la mission de service public. Si tel n'est pas le cas, les dérogations ne pourront être mises en œuvre et les règles classiques du droit de l'Union s'appliqueront.

Dans trois cas de figure, le droit de l'Union a permis de sanctionner des situations touchant à la desserte maritime de la Corse. Dans ces trois cas de figure, aucune dérogation n'a été acceptée. Il s'agit, en premier lieu, de la réponse de l'opérateur maritime à un appel d'offres pour un contrat de service public (1), en deuxième lieu, du versement

18. Laurent Sermet, "La notion juridique de l'ultrapériphéricité communautaire", Europe, $\mathrm{n}^{\circ} 6,2002$, p. 3 et suiv.

19. Il s’agit de la Guadeloupe, de la Guyane, de la Martinique, de La Réunion, des Açores, de Madère et des Canaries. 
de "surcompensations" de service public (2) et, en troisième lieu, des protections accordées à la SNCM (3).

\section{La réponse à l'appel d'offres}

Le premier problème posé par la desserte maritime de la Corse au regard du droit de l'Union européenne concerne le comportement de la SNCM dans une affaire particulière, "d'autant plus notable qu'elle ne [concerne] pas, comme à l'accoutumée, une pratique d'entente anticoncurrentielle mais le comportement abusif d'une entreprise en position dominante et son incidence sur la procédure de publicité et de mise en concurrence ${ }^{20}$ ».

En mai 2006, l'Office des transports de la Corse (OTC), établissement public de la collectivité territoriale de Corse en charge notamment de la desserte maritime de l'île, a lancé une procédure publique pour l'attribution de contrats de service public correspondant à cinq liaisons maritimes entre le port de Marseille et différents ports de Corse, pour la période 2007-2013. Après que quatre offres furent déposées ${ }^{21}$, l'OTC a entamé des négociations avec la SNCM pour l'ensemble des cinq lignes et avec la société Corsica Ferries pour les seules lignes de Balagne et Porto-Vecchio. Les concurrents évincés ainsi que Corsica Ferries ont en conséquence engagé des procédures devant le juge administratif et le conseil de la concurrence ${ }^{22}$.

20. Voir Gabriel Eckert, "Délégation de service public et abus de position dominante », Contrats-Marchés publ. février 2007, $\mathrm{n}^{\mathrm{o}} 2$, comm. 54.

21. Voir Cons. conc., déc. $\mathrm{n}^{\mathrm{0}} \mathrm{06}-\mathrm{MC}-03,11$ déc. 2006 suite à l'appel d'offres, plusieurs propositions ont été remises le 4 août 2006, date limite de remise des offres: une offre globale de la SNCM portant sur l'ensemble des lignes; une offre de la société Corsica Ferries comportant différentes options et portant alternativement sur les lignes Marseille-Bastia, Marseille-Ajaccio, Marseille-Balagne, Marseille-Propriano et Marseille-Porto-Vecchio; une offre de la CMN portant sur 6 propositions individuelles différentes, s'excluant mutuellement et comprenant soit des demiservices permanents, soit des services complets, permanents et supplémentaires; et, enfin, une offre d'un groupement momentané constitué par la société Corsica Ferries et de la CMN portant alternativement soit sur les lignes Marseille-Bastia et Marseille-Propriano, soit sur les lignes Marseille-Ajaccio et Marseille-Propriano.

22. Sur demande de la société Corsica Ferries et de la CMN, le juge des référés précontractuels du tribunal administratif de Bastia a, par une ordonnance du 23 octobre 2006, suspendu la procédure de passation et enjoint à la collectivité de procéder à un nouvel examen de l'offre présentée par le groupement composé de la $\mathrm{CMN}$ et de Corsica Ferries, sans toutefois faire droit à la demande de cette dernière société tendant d'une part à réintégrer sa candidature individuelle pour les 3 lignes de Bastia, Ajaccio et Propriano et d'autre part d'écarter la SNCM des négociations. 
Les réclamations devant l'ancêtre de l'Autorité de la concurrence étaient fondées sur deux séries de griefs et en particulier sur le fait que la SNCM avait commis un abus de position dominante en déposant une offre globale portant de manière indivisible sur les cinq lignes faisant l'objet de la mise en concurrence ${ }^{23}$. Dans sa réponse aux demandes de mesures conservatoires du 11 décembre $2006^{24}$, le Conseil de la concurrence, se fondant à la fois sur les articles du Code de commerce et des traités européens, considère que la SNCM a bien commis un abus dans la mesure où «l'effet d'éviction de l'offre présentée par la SNCM est encore plus incontestable dans la mesure où d'une part, le recours - au moins partiel - au service de cette société est incontournable pour la collectivité, faute pour les autres concurrents de pouvoir formuler des offres portant sur l'ensemble des lignes, et d'autre part, le refus de la SNCM de s'engager de manière ferme sur le montant de la subvention demandé ligne par ligne interdit à l'office la possibilité même de comparer les résultats de la compétition ${ }^{25}$ ".

À ce sujet, le juge administratifif ${ }^{26}$, saisi par un concurrent, a considéré l'OTC avait commis une faute en engageant la négociation avec un opérateur économique dont l'offre n'était pas accompagnée de tous ces documents ou renseignements ${ }^{27}$.

Ces décisions du Conseil de la concurrence sanctionnant l'opérateur public et du Conseil d'État sanctionnant l'autorité organisant la mise en concurrence ont imposé la reprise intégrale de la procédure de

23. En premier lieu, les requérants estimaient qu'il existait une entente entre la SNCM et la collectivité publique délégante. Cependant, le Conseil de la concurrence se déclare incompétent pour examiner ce premier grief dans la mesure où un tel comportement n'est pas détachable de la procédure de passation du contrat administratif.

24. Voir Cons. conc., déc. $\mathrm{n}^{0}$ o6-MC-03, 11 déc. 2006.

25. Voir le point 124 de la décision. On soulignera que le Conseil parvient à cette conclusion alors même qu'il a explicitement considéré que «le dépôt d'une offre globale, qu'il soit autorisé, voire encouragé, ou non, par l'entreprise ou la collectivité organisant la compétition, ne peut être considéré comme étant, par nature, abusif dès lors qu'il émane d'une entreprise en position dominante », point 113.

26. CE, sect., 15 déc. 2006, Société Corsica Ferries, ${ }^{\circ} 298618$.

27. Pour le juge, il n'est possible d'entamer les négociations «que si cette insuffisance, d'une part, ne fait pas obstacle à ce que soit appréciée la conformité de l'offre aux exigences du cahier des charges et, d'autre part, n'est pas susceptible d'avoir une influence sur la comparaison entre les offres et le choix des candidats qui seront admis à participer à la négociation ». 
passation $^{28}$, mais confirment surtout la jurisprudence issue de la décision du tribunal des conflits, Aéroports de Paris en date du 18 octobre $1999^{29}$, selon laquelle, d'une part, les actes par lesquels les personnes publiques font usage, pour l'organisation du service public dont elles ont la charge, de prérogatives de puissance publique doivent être conformes au droit de la concurrence pour pouvoir être légaux, et, d'autre part, les activités des mêmes personnes publiques, intervenant dans la sphère économique, qui sont détachables de leurs actes de puissance publique, peuvent être qualifiées d'ententes et d'abus de position dominante.

\section{L'octroi de surcompensation de service public}

Un deuxième problème est apparu dans le cadre de la DSP pour la desserte maritime de la Corse pour la période 2007-2013. La convention portant sur la fourniture de services maritimes réguliers sur les lignes entre le port de Marseille et les ports de Bastia, Ajaccio, Porto-Vecchio, Propriano et Balagne distingue le service permanent "passager et fret » que le groupement SNCM-CMN doit assurer de manière régulière pendant toute l'année et le service complémentaire de renforcement des prestations au profit des passagers à fournir pour les pointes de $\operatorname{trafic}^{30}$. Il s'agit de deux services différents, mais qui relèvent tous les deux de l'activité encadrée par les autorités publiques pour répondre aux besoins de continuité territoriale, car chacun de ces services permet à celui qui en assure la prestation de percevoir des compensations financières ${ }^{31}$.

Saisie par une plainte de Corsica Ferries, la Commission de l'Union européenne a étudié chaque service et est arrivée à la conclusion que, si la subvention accordée en compensation du service permanent ou service de base entre Marseille et la Corse est légale, en revanche celle attribuée pour les prestations de service complémentaire ne correspondent pas aux critères de la compensation de service public posés par

28. Cette nouvelle procédure a également été attaquée, mais le Conseil d'État a jugé qu'elle ne comportait pas d'illégalité (CE, 13 juil. 2012, Compagnie méridionale de navigation, Société nationale corse méditerranée, $\mathrm{n}^{\text {os }} 355616,355622,358396$.

29. T. confl., 18 oct. 1999, $\mathrm{n}^{\circ}$ 03174, publié au recueil Lebon.

30. Comm. CE, déc. $\mathrm{n}^{\circ}$ 2013/435/UE, 2 mai 2013 concernant l'aide d'État SA.22843 (2012/C) (ex 2012/NN) mise à exécution par la France en faveur de la SNCM et de la CMN, JOUE L 220, p. 20.

31. L'art. 3 de la CDSP précise que la DSP ne donne pas lieu à l'exclusivité sur les lignes considérées, mais donne la possibilité à d'autres compagnies d'effectuer un service régulier sans compensation financière. Néanmoins, un nouvel entrant éventuel serait soumis à des obligations, présentées dans la décision d'ouverture. 
la jurisprudence Altmark ${ }^{32}$ qui, pour mémoire et en substance, fixe le cadre juridique dans lequel les personnes publiques peuvent verser des subventions publiques à une entreprise en contrepartie de la réalisation d'un service public sans tomber dans le régime des aides d'État ${ }^{33}$.

En l'espèce, la Commission dénie la qualification de service d'intérêt économique général pour le service complémentaire. À cet égard, elle souligne notamment que "concernant les horaires, tandis que la définition du service de base imposait un horaire de départ minimal et une plage d'arrivée stricte, la définition du service complémentaire ne stipulait aucun horaire à respecter ». "Concernant la fréquence, aucune obligation n'est imposée s'agissant du service complémentaire par le cahier des charges de la CDSP, la capacité de transport étant définie annuellement sur les périodes de pointe. En outre, l'exploitation de services organisés selon une distinction similaire a été menée avec des navires et des équipages distincts sur la période 2002-2006 ${ }^{34}$.»

Par cette décision, la Commission a condamné la SNCM à rembourser les compensations qu'elle a reçues au titre du service complémentaire de la $\operatorname{CDSP}^{35}$. La France, n'ayant pas entrepris de récupérer ces aides

32. CJCE, 24 juil. 2003, Trans GmbH et Regierungspräsidium Magdeburg c/ Nahverkehrsgesellschaft Altmark GmbH, C-280/oo, Rec. 2003, p. 7747.

33. Le droit de l'Union ne s'oppose pas à la mise en place ou à la subvention de SIEG, mais détermine 4 conditions de transparence qui doivent être remplies pour que ces subventions ne soient pas considérées comme des aides d'État et soient ainsi soustraites à l'obligation de notification à la Commission : 1) les obligations de service public doivent être clairement définies; 2) la compensation doit être préalablement calculée de façon objective et transparente, afin d'éviter qu'elle comporte un avantage économique susceptible de favoriser l'entreprise bénéficiaire par rapport à des entreprises concurrentes; 3) la compensation ne saurait dépasser ce qui est nécessaire pour couvrir tout ou partie des coûts occasionnés par l'exécution des obligations de service public en tenant compte des recettes y relatives ainsi que d'un bénéfice raisonnable relatif à l'exécution de ces obligations ; 4) enfin, lorsque le choix de l'entreprise n'est pas effectué dans le cadre d'une procédure de marché public permettant de sélectionner le candidat capable de fournir ces services au coût économiquement le plus avantageux (le «mieux disant») pour la collectivité, le niveau de la compensation nécessaire doit être déterminé sur la base d'une analyse des coûts qu'une entreprise moyenne, bien gérée et adéquatement équipée en moyens de transport, aurait encourus pour exécuter ces obligations.

34. Comm. CE, déc. $n^{\circ}$ 2013/435/UE, 2 mai 2013, point 141.

35. Le montant d'aide à récupérer inclut les éléments suivants: (a) le montant des compensations effectivement versées de 2007 à 2011 au titre du service complémentaire qui s'élève à 172,744 millions d'euros; (b) les acomptes versés mensuellement pour l'année 2012 au titre du service complémentaire, estimés actuellement à 38 millions d'euros ainsi que le reliquat de la compensation, qui doit être versé après transmission du rapport définitif d'exécution des services, si ce dernier a déjà 
illégales incompatibles avec le marché commun, a été sanctionnée pour manquement par la Cour de justice en juillet $2015^{36}$.

Cette affaire illustre une des principales difficultés rencontrées par les services publics en France. Jusqu'à présent la simple qualification d'une activité d'intérêt général suffisait à la faire échapper aux règles de droit commun. Désormais, il faut encore démontrer que l'application de règles spécifiques est justifiée, c'est-à-dire qu'elles sont nécessaires pour assurer l'accomplissement de la mission particulière qui a été impartie aux entreprises à qui l'on a confié la prestation du service public ${ }^{37}$.

\section{Les protections accordées à la SNCM}

Un troisième problème a été rencontré par la SNCM, mais cette fois-ci sans que son comportement puisse être incriminé. La CTC avait décidé, par une délibération en date du 9 novembre 2012, de mettre en place un nouveau régime d'obligations de service public pour les liaisons de transport maritime de passagers et de marchandises entre les ports continentaux de Marseille, Toulon ou Nice et les ports de Corse. Plus précisément, il était question de remplacer le régime de déclaration existant par un régime d'autorisation préalable qui obligeait toute compagnie désirant assurer des liaisons entre les ports de Corse et ceux du continent à soumettre un projet à l'Office des transports

été versé; (c) les acomptes versés mensuellement pour l'année 2013 au titre du service complémentaire jusqu'à la date de la présente décision, estimés actuellement à 9,5 millions d'euros, étant rappelé que la France doit annuler tous les versements postérieurs à cette date.

36. Voir CJUE, 9 juil. 2015, Commission européenne c/ République française, C-63/14, ECLI :EU :C :2015 :458: «En n'ayant pas pris, dans les délais prescrits, toutes les mesures nécessaires afin de récupérer auprès de la Société nationale maritime Corse-Méditerranée (SNCM) SA les aides d'État déclarées illégales et incompatibles avec le marché intérieur par l'article 2, paragraphe 1, de la décision 2013/435/UE de la Commission, du 2 mai 2013, concernant l'aide d'État SA.22843 (2012/C) (ex 2012/NN) mise à exécution par la France en faveur de la Société Nationale Corse Méditerranée et la Compagnie Méridionale de Navigation, en n'ayant pas annulé, dans les délais prescrits, tous les versements des aides visées à cet article 2, paragraphe 1, et en n'ayant pas informé la Commission européenne, dans le délai imparti, des mesures prises pour se conformer à cette décision, la République française a manqué aux obligations qui lui incombent en vertu de l'article 288 , quatrième alinéa, TFUE et des articles 3 à 5 de ladite décision. »

37. Dans ce sens voir en particulier, CJCE, 19 mai 1993, Procédure pénale c/ Paul Corbeau, C-320/91, Rec. 1993, p. I-02533. 
de Corse et à signer une convention annuelle ${ }^{38}$. Pour les compagnies intéressées, le nouveau régime favorise les délégataires du service public soit, par conséquent, la $\mathrm{SNCM}^{39}$ et leur permet surtout d'être protégés de la concurrence que représentent les autres compagnies maritimes, tout ceci en limitant leur liberté de prestation de service reconnue par le règlement européen sur le cabotage maritime ${ }^{40}$.

Pour bien comprendre l'enjeu de cette affaire, il faut rappeler que l'article 4 de ce règlement offre aux autorités nationales la possibilité d'adopter des mesures en vue de protéger les missions d'intérêt général. Ainsi, « un État membre peut conclure des contrats de service public avec des compagnies de navigation qui participent à des services réguliers à destination et en provenance d'îles ainsi qu'entre des îles ou leur imposer des obligations de service public en tant que condition à la prestation de services de cabotage ». "Lorsqu' un État membre conclut des contrats de service public ou impose des obligations de service public, il le fait sur une base non discriminatoire à l'égard de tous les armateurs communautaires. » Le droit de l'Union précise que «s'ils imposent des obligations de service public, les États membres s'en tiennent à des exigences concernant les ports à desservir, la régularité, la continuité, la fréquence, la capacité à prester le service, les tarifs pratiqués et l'équipage du navire». "Toute compensation due, le cas échéant, en contrepartie d'obligations de service public doit être versée à tous les armateurs communautaires. »

Saisi une nouvelle fois par les concurrents de la SNCM, le tribunal administratif de Bastia a jugé que la délibération du 9 novembre 2012 était contraire au règlement de 1992, considérant «que la Collectivité territoriale de Corse [n'avait pas prouvé] que le régime d'autorisation préalable [...] [était] rendu nécessaire par l'existence d'un besoin réel de service public résultant de l'insuffisance des services de transports réguliers ${ }^{41}$ ".

38. La conclusion de cette convention a pour conséquence d'obliger les compagnies contractantes à respecter la grille tarifaire mise en place par la CTC, laquelle impose un tarif résident qui est le même que celui prévu par la délégation de service public, un autre tarif plancher pour les passagers non résidents qui sera fixé par référence aux tarifs fixés pour la délégation de service public et un tarif plancher pour le transport de fret roulant qui est égal au tarif plafond de la délégation de service public.

39. En effet, celle-ci se trouve en situation de pratiquer des prix systématiquement inférieurs à ceux de ses concurrents, tout en bénéficiant de subventions publiques.

40. Cons. UE, règl. $\mathrm{n}^{\mathrm{o}}$ 3577/92, 7 déc. 1992, art. $1^{\mathrm{er}}$.

41. TA Bastia, 17 oct. 2013, Corsica Ferries France et CGPME de Corse c/ C.T.C., $\mathrm{n}^{\mathrm{os}} 1300012$ et 1300014 . 
Ici, c'est bien l'autorité publique qui est sanctionnée pour ne pas avoir respecté le droit de l'Union et les règles du marché intérieur définies par le règlement sur le cabotage maritime. Néanmoins, on trouve le même écueil que celui relevé précédemment. En effet, la liberté des collectivités publiques dans l'organisation d'un service public n'est pas totale. Elles ne peuvent intervenir que si «l'application [des règles européennes] ne fait pas échec à l'accomplissement en droit ou en fait de la mission [de service public] ». À défaut, il leur faut laisser les lois du marché faire.

$\mathrm{Au}$ terme de ce rapide panorama, la desserte maritime de la Corse semble toujours pouvoir être assurée malgré l'évolution du cadre juridique sous l'influence du droit de l'Union européenne. Si la SNCM apparaît comme une compagnie maritime qui s'est trouvée, très souvent, prise au piège des règles du marché intérieur ou de celles de la concurrence, c'est avant tout parce que cette entreprise de service public, comme les autorités publiques, ne se s'est pas préparée à ces évolutions juridiques. Toutefois, il faut reconnaître que les contentieux ne portent plus, aujourd'hui, sur l'organisation de la desserte maritime, preuve s'il en est que ses acteurs se sont définitivement adaptés pour se conformer au droit de l'Union européenne.

Maître de conférences en droit public CRDEI, Centre d'excellence Jean Monnet d'Aquitaine Université de Bordeaux 